“ (C) 2013 IEEE. Personal use of this material is permitted. Permission from IEEE must be obtained for all other uses, in any current or future media, including

reprinting/republishing this material for advertising or promotional purposes, creating new collective works, for resale or redistribution to servers or lists, or reuse of any copyrighted component of this work in other works." 


\section{A Method to Widen the Scattering Bandwidth of Closed Cylindrical Active Coated Nano Particles}

\author{
Junping Geng*(1), Ronghong Jin ${ }^{(1)}$, Xianling Liang ${ }^{(1)}$ \\ (1) Electronic Engineering Department, \\ Shanghai Jiao Tong University, \\ Shanghai, China \\ gengjunp@gmail.com
}

\begin{abstract}
Plane wave scattering from two closely spaced, closed, active, cylindrical coated nano particles (C-CNPs), which have slightly different resonance frequencies, is studied numerically. Although the distance between them is only $0.4 \lambda$, the scattering cross-section(SCS) background value of this two-C-CNP system is increased $5 \mathrm{~dB}$ when $\mathrm{r} 2=15.1 \mathrm{~nm}$ and the $3 \mathrm{~dB}$ SCS bandwidth is increased to $150 \mathrm{GHz}$, which is a substantially wider working bandwidth near the SCS peak than the one associated with either single C-CNP, i.e., 90 GHz.
\end{abstract}

Keywords- active coated nano particles; gain media; nanometer antennas; nanotechnology

\section{INTRODUCTION}

Nano- structures and particles incorporating metals have shown attractive prospects for applications in the areas of biology, medicine [1-3], efficient solar cells, high resolution microscopy, optical communications [4-6], and sensor technologies. Because of their surface plasmonic character, metals at optical frequencies behave as epsilon negative (ENG) media. By combining them with dielectric media doped, e.g., with rare earth ions, these nano-sized plasmonic particles can be used to achieve highly sub-wavelength resonators and lasing elements [7-13].

Active cylindrical nano antenna systems show very attractive performance characteristics. In [11-13], the active closed cylindrical nano-antenna was studied. It exhibits both dual polarization and very good radiated power behavior. While these basic nano-structures produce excellent scattering and absorption properties, their operating bandwidths are very narrow, a property which blocks many potential applications. Other more complex structures could be formed with current nano-fabrication processes and could exhibit more bandwidth and other desirable performance characteristics. In this paper, two active nanometer antennas, which are only slightly different, are introduced in close proximity to each other. This configuration is studied numerically; it provides a path to wider working bandwidths.

\author{
Richard W. Ziolkowski ${ }^{(2,3)}$ \\ (2) Electrical and Computer Engineering Department \\ University of Arizona, Tucson, AZ, USA \\ (3) College of Optical Sciences, \\ University of Arizona, Tucson, AZ, USA
}

\section{TWO ACTIVE NANOMETER ANTENNAS WITH SMALL STRUCTURE DIFFERENCES}

The two active cylindrical coated nanoparticle (C-CNP) configuration which was investigated is shown in Fig.1. The annular shell and the top and bottom spacers are silver, whose properties are described by a Drude model [11]. The long axes of the two C-CNPs are parallel and their ends sit on a lossless silica substrate. The gain impregnated filling lies in the center of the nano-structures. This medium is taken to be lossless silica for the passive nano-antenna comparison cases. The gain material for the active nano-antenna was selected following [11], which was silica doped with rare earth (erbium $\mathrm{Er}^{3+}$ ) ions or quantum dots. It obeys the Lorentz model for the relative permittivity:

$$
\varepsilon_{r}(\omega)=\varepsilon_{\infty}+\frac{\left(\varepsilon_{S}-\varepsilon_{\infty}\right) \omega_{0}^{2}}{\omega_{0}^{2}+j \omega \Gamma-\omega^{2}}
$$

For a gain impregnated $\mathrm{SiO}_{2}$ core, if $\kappa=-0.25$ at the specified resonance frequency, $\mathrm{f}_{0}=600 \mathrm{THz}$, the resulting $\varepsilon_{\mathrm{r}}$ values are shown in Fig. 1 for the value of the damping frequency $\Gamma=$ $10^{-3} \omega_{0}, \omega_{0}$ being the angular resonance frequency

\section{SIMULATION RESULTS}

The CNP type of nano-structure (basically a core-shell structure) has been studied by many researchers, e.g., $[9,10]$. Its parameters and characteristics have been investigated thoroughly. As in [11] and as noted above, the CNP is filled with gain impregnated $\mathrm{SiO}_{2}$. The source is a linearly polarized plane wave whose electric field vector is oriented along the axis of the cylinders. The coated film is silver and has a thickness equal to $6 \mathrm{~nm}$. The height of the core is $l=34.5 \mathrm{~nm}$. The radii of the cores are $\mathrm{r} 1$ and $\mathrm{r} 2$, respectively, for particle 1 and particle 2. As in [13], we choose both $\mathrm{r} 1$ and $\mathrm{r} 2$ to be close to $15 \mathrm{~nm}$. The distance between the two particles is $2 \mathrm{rc}$. We chose it to be $2 \mathrm{rc}=200 \mathrm{~nm}$, which is equal to $0.4 \lambda$ for the $600 \mathrm{THz}$ excitation frequency (500 $\mathrm{nm}$ wavelength).

Here, we give an example with $\mathrm{r} 1=15 \mathrm{~nm}$. The value of $\mathrm{r} 2$ is then swept through several values, and the corresponding scattering cross section values were obtained. The simulated responses for these configurations are shown in Fig.2. The

This work was supported in part by contracts 2009CB320205, 60821062, 10ZR1416600 and 20090073120033 and by NSF contract number ECCS-1126572 
SCS background of the two active particle-CNP system is increased $5 \mathrm{~dB}$ when $\mathrm{r} 2=15.1 \mathrm{~nm}$. Furthermore, the $3 \mathrm{~dB}$ SCS bandwidth is $150 \mathrm{GHz}(599.95-600.1 \mathrm{THz})$. This is a wider working bandwidth near the SCS peak value than the bandwidth of a single particle-CNP: $90 \mathrm{GHz}$ (600.01-600.1 $\mathrm{THz})$.

\section{CONCLUSIONS}

The plane wave excitation of two nearby, parallel, slightly different, active cylindrical coated nano particles (C-CNPs) was investigated. It was found that with an optimized spacing, a $5 \mathrm{~dB}$ increase of the SCS background value and a wider $3 \mathrm{~dB}$ SCS bandwidth is obtainable. These are significant enhancements over the corresponding single $\mathrm{C}-\mathrm{CNP}$ values.

\section{REFERENCES}

[1] Braulio García-Cámara1, etc., " Light scattering by an array of electric and magnetic nanoparticles," Opt. Exp., vol. 18, pp.10001, May 2010.

[2] P. K. Jain, etc., "Noble metals on the nanoscale: optical and photot ermal properties and some applications in imaging, sensing, biology, and medicine," Acc. Chem. Res., vol.41, pp.1578-1586, 2008.

[3] X. Liu, and Q. Huo, "A washing-free and amplification-free one-step homogeneous assay for protein detection using gold nanoparticle probes and dynamic light scattering," J. Immunol. Methods, vol. 349, No.1-2, pp. 38-44, 2009.

[4] K. R. Catchpole, and A. Polman, "Plasmonic solar cells," Opt. Exp., vol.16, pp. 21793-21800, 2008.

[5] S. Kawata, Y. Inouye, and P. Verma, "Plasmonics for near-field nanoimaging and superlesing," Nat. Photonics, vol. 3, pp.388-394, 2009.

[6] M. Guillaumée, etc., Polarization sensitive silicon photodiodes using nanostructured metallic grids," Appl. Phys. Lett., vol. 94, 193503, 2009.

[7] J. A. Gordon and R. W. Ziolkowski, "Colors generated by tunable plasmon resonances and their potential application to ambiently illuminated color displays," Solid State Commun., vol. 146, pp. 228-238, Apr. 2008.

[8] J. A. Gordon and R. W. Ziolkowski, "The design and simulated performance of a coated nano-particle laser," Opt. Exp., vol. 15, pp. 2622-2653, March 2007.

[9] J. A. Gordon and R. W. Ziolkowski, "CNP optical metamaterials," Opt. Exp, vol. 16, pp. 6692-6716, April 2008.

[10] J. Geng, R. W. Ziolkowski, R. Jin, X. Liang, "Numerical study of the near-field and far-field properties of active open cylindrical coated nanoparticle antennas," IEEE Photonics J., vol.3, pp.1092-1110, Dec., 2011.

[11] J. Geng, R. W Ziokowshi, S. Cambell, R. Jin, X. Liang, "Studies of nanometer antennas incorporating gain material using CST," Proc. 2011 IEEE International Symposium on Antennas and Propagation, 3 -8 July 2011, Spokane, USA.

[12] J. Geng, R. W Ziokowshi, R. Jin, X. Liang, "Study on the dual polarizations of the active cylindrical coated nano-particle antennas," iWAT2012, Tucson, USA, March 2012.

[13] J. Geng, R. Jin, X. Liang and R. W. Ziolkowski, "Active cylindrical coated nano-particle antennas: Polarization-dependent scattering properties," J. Electromagnet. Wave (JEMWA), submitted Jan. 2013

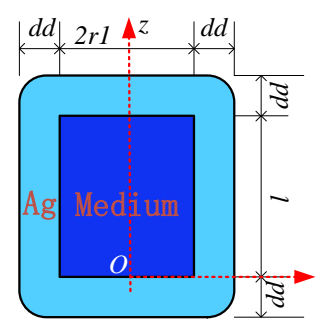

(a)

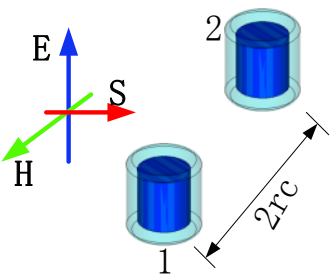

(b)
Fig.1 CST Model. (a) Parameters of a single closed active cylindrical coated nano particle, and (b) isometric view of the two parallel, slightly different, active nano particles excited by a vertically polarized plane wave.

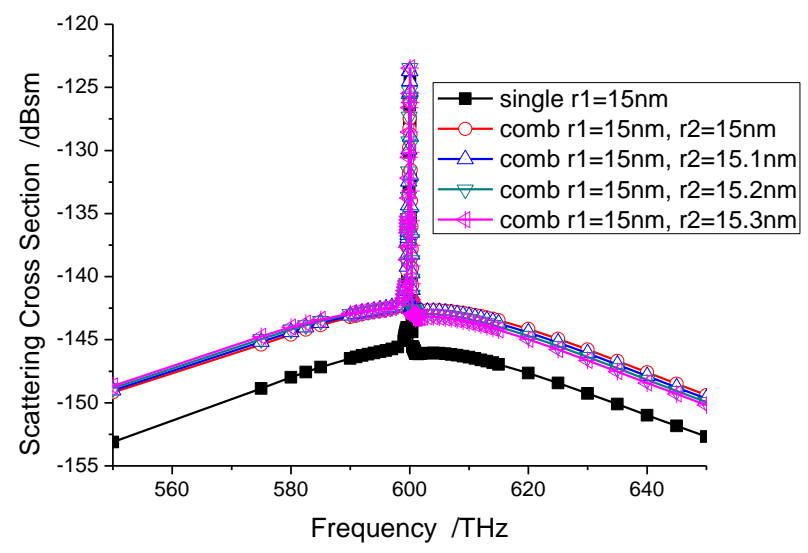

(a)

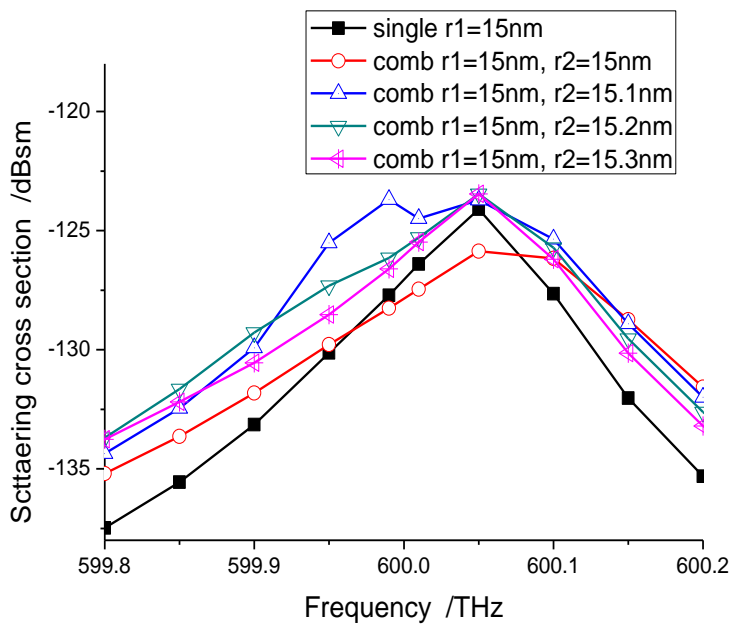

(b)

Fig.2 Scattering cross sections versus frequency for plane wave scattering from two parallel, active cylindrical coated nano particles having slight differences in their geometries when $\mathrm{r} 2$ is swept, $\mathrm{r} 1=15 \mathrm{~nm}$ is fixed, and the medium is low loss with $\Gamma=10^{-3} \omega_{0}$ (a) SCS over the whole frequency band, and (b) zoom-in view near the maximum response. 